\title{
NOUVELLE
}

\section{L'imagerie fonctionnelle des neurones individuels au sein de réseaux complexes}

Romuald Nargeot ${ }^{1}$, Guillaume Radecki ${ }^{2}$, lleana 0. Jelescu ${ }^{2}$, Denis Le Bihan², Luisa Ciobanu

\author{
${ }^{1}$ Université de Bordeaux et CNRS, Institut de neurosciences \\ cognitives et intégratives d'Aquitaine, UMR 5287, \\ F-33000 Bordeaux, France ; \\ ${ }^{2}$ NeuroSpin, Commissariat à l'énergie atomique \\ et aux énergies alternatives, \\ 91191 Gif-sur-yvette, France. \\ luisa.ciobanu@cea.fr
}

Premières images de neurones en IRM fonctionnelle

Comprendre l'origine des comportements normaux ou pathologiques nécessite d'analyser les propriétés fonctionnelles des réseaux de neurones du système nerveux central (SNC) et leur régulation par les entrées sensorielles périphériques. L'analyse de ces phénomènes, initialement basée sur des circuits simples $[1,2]$, a montré la grande diversité des neurones qui, par leurs propriétés membranaires et synaptiques, contribuent ensemble à organiser et à émettre les actes moteurs. La diversité neuronale est d'autant plus importante que les comportements sont complexes. Ainsi, les comportements motivés ou dirigés vers un but tels que les comportements alimentaires, sexuels ou sociaux, sont produits par des structures cérébrales complexes, composées de nombreux neurones aux propriétés fonctionnelles variées, qui rendent difficile une analyse cellulaire [3]. Pourtant, cette étude à l'échelle des cellules individuelles est fondamentale, non seulement pour appréhender le fonctionnement normal du SNC, mais également pour comprendre les mécanismes intimes des dérèglements à l'origine des conduites pathologiques.

Les techniques d'imagerie fonctionnelle ont permis des progrès considérables dans la visualisation du fonctionnement normal et pathologique du SNC. Ainsi, l'imagerie par résonance magnétique nucléaire (IRM) est aujourd'hui largement utilisée dans le contexte d'études expérimentales et cliniques. La plupart de ces études sont limitées par une résolution spa- tiale de l'ordre de $(0,3 \mathrm{~mm})^{3}$, une échelle qui permet de visualiser des structures nerveuses de plusieurs centaines de cellules, mais sans en distinguer les neurones constitutifs. La microscopie par résonance magnétique (MRM) permet cependant d'atteindre des résolutions de l'ordre du micromètre $[4,5]$. Pourtant, jusqu'à présent, la vaste majorité des études en MRM se sont limitées à l'imagerie de structures statiques plutôt que fonctionnelles. Depuis une dizaine d'années, une nouvelle technique d'IRM fonctionnelle a été introduite : I'IRM rehaussée au manganèse (MEMRI). MEMRI utilise I'ion manganèse $\left(\mathrm{Mn}^{2+}\right)$ comme marqueur de l'activité neuronale [6]. Cet analogue du calcium s'accumule dans les neurones en fonction de leur activité électrique individuelle, en empruntant les canaux calciques et cationiques de leur membrane plasmique. Grâce à ses propriétés paramagnétiques, le $\mathrm{Mn}^{2+}$ modifie localement l'environnement magnétique des protons, permettant ainsi de visualiser cet effet sur certaines images IRM.

L'étude MEMRI que nous avons menée a permis de faire passer la MRM de l'observation de structures statiques à l'observation des propriétés fonctionnelles dynamiques des réseaux neuronaux et de neurones individuels. Pour cela, nous avons utilisé le mollusque marin aplysie (Figure 1), dont le système nerveux est composé d'un nombre réduit de neurones qui sont les plus gros du règne animal (quelques centaines de micromètres) [7]. Chez ce modèle animal simple, les neurones formant les réseaux responsables de la genèse de son comportement alimentaire ont été identifiés au sein des ganglions buccaux par des méthodes d'électrophysiologie et d'histologie, et offrent ainsi une opportunité unique pour une analyse en IRM fonctionnelle [8-10].

Accumulation in vivo de $\mathrm{Mn}^{2+}$ dans des neurones identifiés

Pour visualiser l'activité neuronale en IRM, une faible concentration de $\mathrm{Mn}^{2+}$ est injectée chez l'animal [7]. Après une période pendant laquelle l'aplysie effectue sa recherche de nourriture sans aucun stimulus extérieur, les ganglions buccaux sont isolés puis analysés par résonance magnétique à ultra-haut champ (17,2 Tesla). Une série d'expériences a permis de vérifier que, comme chez les

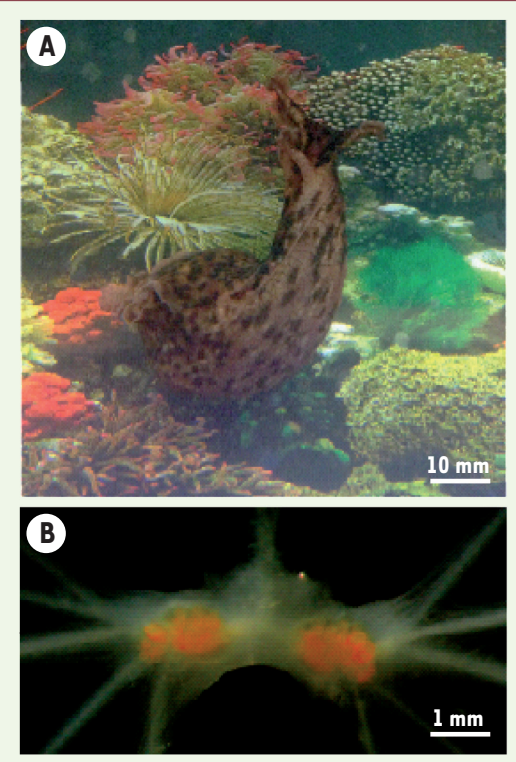

Figure 1. Photographies d'une aplysie (Aplysia californica) (A) et de ganglions buccaux isolés (B). 


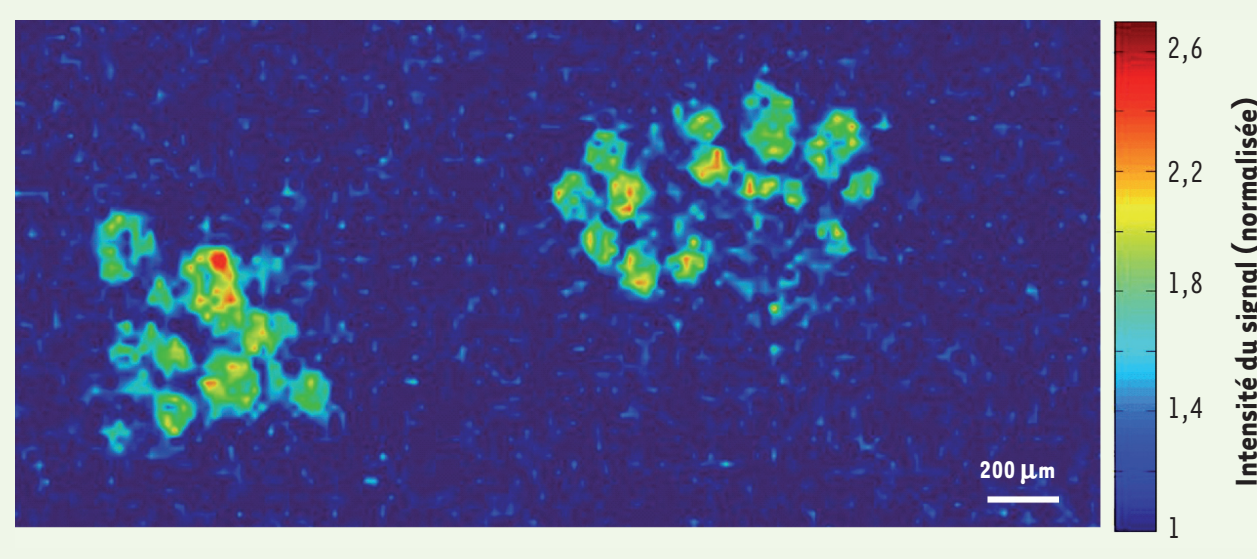

Figure 2. Image IRM des ganglions buccaux d'une aplysie stimulée par un aliment et montrant l'accumulation intracellulaire de $\mathrm{Mn}^{2+}$. Les régions hyperintenses représentent des neurones individuels. L'intensité du signal (normalisée à l'intensité du signal de l'eau) est proportionnelle à la concentration intracellulaire de $\mathrm{Mn}^{2+}$ et donc à l'activité neuronale. Résolution spatiale : $25 \mu \mathrm{m}$.

mammifères, le $\mathrm{Mn}^{2+}$ s'accumule dans les réseaux neuronaux. Cette accumulation est visualisée par une intensification du signal sur les images IRM. Cette méthode établit que les zones d'accumulation $\mathrm{du} \mathrm{Mn}^{2+}$ correspondent précisément aux somas des neurones moteurs identifiés individuellement et dont l'activité électrique est responsable des contractions musculaires exécutées lors de la recherche de nourriture. II est intéressant de noter que cette accumulation de $\mathrm{Mn}^{2+}$ varie d'un neurone à l'autre proportionnellement à la durée et à l'intensité de l'activité électrique résultant de l'ouverture, dépendante du voltage, des canaux membranaires propres à chaque neurone. Ces résultats, obtenus chez des animaux non stimulés, montrent que la concentration en $\mathrm{Mn}^{2+}$ dans les neurones reste relativement faible (environ $20 \mu \mathrm{M}$ ).

\section{Modifications de l'activité neuronale par des stimulus sensoriels}

Pour stimuler leur système nerveux, les animaux sont mis en présence d'aliments. Ces aliments activent des récepteurs sensoriels externes (péri-oraux) et internes (intra-buccaux) qui déclenchent les phases d'excitation et de consommation du comportement alimentaire. La phase d'excitation (ou appétitive) correspond à une intensification du déplacement de l'animal, qui recherche sa nourriture mais ne modifie pas drastiquement ses mouvements buccaux. La phase de consommation correspond en revanche à l'inges- tion de l'aliment. Elle se différencie de la précédente par l'arrêt des déplacements et une intensification/répétition des mouvements buccaux. Ainsi, à la fois l'origine des stimulus sensoriels et les réponses motrices évoquées distinguent ces deux phases du comportement. Chez les animaux stimulés par l'aliment et ayant préalablement reçu une injection de $\mathrm{Mn}^{2+}$, l'imagerie des ganglions buccaux met en évidence une très nette augmentation du signal dans les neurones moteurs responsables des mouvements buccaux (Figure 2). Cette augmentation du signal correspond à une concentration intracellulaire de $\mathrm{Mn}^{2+}$ d'environ $64 \mu \mathrm{M}$.

\section{Réponses différentielles des neurones}

Les effets différentiels des stimulus externes et internes peuvent être observés : la très forte augmentation d'intensité du signal dans les neurones d'animaux qui ont été à la fois excités (stimulation externe) et nourris (stimulation interne) ne s'observe pas chez des animaux qui n'ont été qu'excités (stimulation externe). Dans ce dernier cas, les concentrations intracellulaires de $\mathrm{Mn}^{2+}$ restent proches, bien que légèrement supérieures $(\sim 30 \mu \mathrm{M})$, de celles observées chez les animaux non stimulés $(\sim 20 \mu \mathrm{M})$. Il est à noter que ces modifications du signal varient selon les neurones, et que l'on met en évidence la présence d'excitations, mais aussi d'inhibitions au sein du réseau. Ainsi, I'IRM fonctionnelle permet de visualiser non seulement des modifications d'activités neuronales individuelles, mais également différents profils de réponses neuronales.

\section{Conclusion et perspectives}

Cette étude [7] offre une preuve de concept de la possibilité d'une imagerie fonctionnelle des neurones individuels au sein de réseaux complexes qui génèrent des fonctions biologiques identifiées. Notons que les concentrations intracellulaires du marqueur d'activité utilisé $\left(\mathrm{Mn}^{2+}\right)$ sont très inférieures à celles qui produisent des effets toxiques pour les cellules. Ainsi, cette avancée technique permet l'analyse du fonctionnement dynamique des neurones dans les conditions physiologiques, et pourra être étendue aux conditions pathologiques. D'autre part, bien qu'actuellement difficile, son application chez les mammifères est envisageable. L'IRM à l'échelle de la cellule sur des tissus fixés porcin et humain a déjà été réalisée [11]. L'amélioration de la technique dans un futur proche devrait permettre d'appliquer cette approche à des tissus vivants chez les mammifères. $\diamond$ The functional imaging of individual neurons within complex networks

\section{LIENS D'INTÉRÊT}

Les auteurs déclarent n'avoir aucun lien d'intérêt concernant les données publiées dans cet article.

\section{REMERCIEMENTS}

Cette étude a bénéficié du soutien financier de l'Agence nationale pour la recherche (ANR13-BSV5-0014-01). 


\section{RÉFÉRENCES}

1. Kandel $\varepsilon R$. The molecular biology of memory storage: a dialogue between genes and synapses. Science $2001 ; 294: 1030-8$

2. Marder $\varepsilon$, Calabrese RL. Principles of rhythmic motor pattern generation. Physiol Rev $1996 ; 76$ : 687-717.

3. Balleine BW, O'Doherty JP. Human and rodent homologies in action control: corticostriatal determinants of goal-directed and habitual action. Neuropsychopharmacol $2010 ; 35$ : 48-69.

4. Ciobanu L, Seeber DA, Pennington CH. 3D MR microscopy with resolution 3.7 microm by 3.3 microm by 3.3 microm. J Magn Reson 2002 ; 158 : 178-82.
5. Ciobanu L, Webb AG, Pennington CH. Magnetic resonance imaging of biological cells. Prog Nucl Magn Reson Spectrosc 2003; $42: 69-93$.

6. Silva AC, Lee JH, Aoki I, Koretsky AP. Manganeseenhanced magnetic resonance imaging (MEMRI): methodological and practical considerations. NMR Biomed $2004 ; 17$ : 532-43.

7. Radecki G, Nargeot R, Jelescu 10, et al. Functional magnetic resonance microscopy at single-cell resolution in Aplysia californica. Proc Natl Acad Sci USA 2014 ; 111 : 8667-72.

8. Nargeot R, Simmers J. Functional organization and adaptability of a decision-making network in Aplysia Front Neurosci 2012 ; 6 : 113
9. Morton DW, Chiel HJ, Cohen LB, Wu JY. Optical methods can be utilized to map the location and activity of putative motor neurons and interneurons during rhythmic patterns of activity in the buccal ganglion of Aplysia. Brain Res 1991 ; 564 : 45-55.

10. Jelescu IO, Nargeot R, Le Bihan D, Ciobanu L. Highlighting manganese dynamicsin the nervous system of Aplysia californica using MEMRI at ultrahigh field. Neuroimage $2013 ; 76: 264-71$.

11. Flint JJ, Lee CH, Hansen B, et al. Magnetic resonance microscopy of mammalian neurons. Neuroimage $2009 ; 46: 1037-40$

\title{
NOUVELL
}

\section{L'investissement parental des espèces animales prédit leur diversité génétique}

\author{
Nicolas Galtier ${ }^{1,2}$, Jonathan Romiguier ${ }^{1,2}$
}

> Si l'on compare la séquence d'ADN d'un de vos chromosomes à celle d'un des chromosomes de votre voisin, on détectera une différence toutes les 5000 paires de bases environ. Si l'on applique la même comparaison à deux individus de l'espèce Drosophila simulans, on enregistrera une différence toutes les 300 paires de bases. La mouche du vinaigre présente une diversité génétique beaucoup plus élevée que celle de notre espèce. Pour quelles raisons? II est remarquable que cette question historique [1] soit restée essentiellement sans réponse jusqu'à très récemment, et cela malgré le rôle central joué par la diversité génétique en biologie des populations et dans les politiques de préservation des espèces [2].

\section{Taille de population et dérive génétique}

La diversité génétique attendue dans une population panmictique - c'est-à-dire sans isolement géographique marqué est proportionnelle au produit de deux paramètres: le taux de mutation et la taille efficace des populations. Le premier de ces deux facteurs se comprend aisément: si un plus grand nombre de mutations apparaissent à chaque génération, la variation génétique augmente. L'autre effecteur majeur, la taille des populations, est sans doute moins intuitif. Il agit non pas sur le taux d'apparition des mutations, mais sur leur durée de vie à l'état polymorphe, plus courte dans les populations à faible effectif. Pour bien le comprendre, il faut réaliser que le devenir d'une mutation neutre (qui ne procure ni avantage ni désavantage à ses porteurs) est incertain, et lié au hasard de la reproduction. Si, par chance, les porteurs d'un allèle mutant venaient à avoir un grand nombre de descendants, la prévalence de cet allèle augmenterait mécaniquement à la prochaine génération - et inversement en cas de faible nombre de descendants. Cet effet aléatoire, appelé dérive génétique, est plus marqué dans les petites que dans les grandes populations: un variant malchanceux peut disparaître en quelques générations d'une population de très petite taille. En raccourcissant la durée de vie des mutations polymorphes, la dérive tend donc à diminuer la diversité génétique dans les petites populations [3].
${ }^{1}$ Institut des sciences de l'évolution, CNRS UMR5554, université Montpellier 2, place $\varepsilon$. Bataillon CC64, 34095 Montpellier, France ;

2 département d'écologie et d'évolution, université de Lausanne, Suisse. nicolas.galtier@univ-montp2.fr

Or, il est communément admis que la taille des populations naturelles est variable dans le temps. La démographie des espèces est affectée par des processus climatiques, écologiques et anthropiques qui font fluctuer leurs abondances. Ainsi, la paléoécologie nous a appris qu'un grand nombre d'espèces européennes étaient confinées il y a 100000 ans, lors du dernier maximum glaciaire, à quelques «zones refuges » (péninsules ibérique, italienne et balkanique) [4]. Ces phases de forte réduction d'effectif, appelées "goulots d'étranglement », ont un impact durable sur la diversité génétique: en intensifiant soudainement la dérive, un goulot fait disparaître de nombreux variants, qui ne se régénéreront que lentement par mutation une fois l'épisode passé. Ces fluctuations temporelles d'effectif sont typiquement invoquées pour expliquer le caractère erratique de la diversité génétique: si elle reflète avant tout le passé des espèces, comment la prédire sur la base des données actuelles? Une synthèse récente n'a pas permis de corréler de manière convaincante la diversité génétique de 167 espèces distinctes à 